\title{
Recyclage de l'urée dans le rumen chez les chèvres laitières : effet du stade physiologique
}

\author{
H Chaoutène, J Brun-Bellut
}

INRA, ENSAIA, 54500 Vandœuvre, France

\begin{abstract}
Summary - Rumen recycled urea in dairy goats: effect of physiological stage. The quantity of urea recycled in the rumen was measured in 4 dry, pregnant or lactating goats. It altered from $50 \mathrm{mg} /$ $\mathrm{kg}$ body weight/d in dry goats to $260 \mathrm{mg}$ in lactating goats, $66 \%$ of the difference being explained by the difference in the quantity of digestible organic matter (MOD) ingested. The quantity of urea recycled, corrected for the MOD ingested, was highest in late pregnancy and during early lactation.
\end{abstract}

Le recyclage de l'urée dans le rumen a été étudié essentiellement chez des ruminants à l'entretien ou en croissance. Nos travaux ont porté sur la mesure des quantités d'urée endogène pénétrant dans le rumen de 4 chèvres laitières à différentes périodes de leur cycle de production : tarissement, gestation et lactation.

Matériel et méthodes - Quatre chèvres alpines chamoisées ( $55 \pm 6 \mathrm{~kg} \mathrm{PV}$ ), adultes, sont utilisées au cours de leur $3^{e}$ lactation. Leur production laitière moyenne est de $2,5 \mathrm{~kg} / \mathrm{j}$. Elles reçoivent une ration composée de foin de prairie permanente $(52 \%$ de la MS), de luzerne $(9 \%)$ et de pulpes de betterave deshydratées $(9 \%)$, d'orge $(17 \%)$ et de tourteaux de soja $(13 \%)$. La ration distribuée contient $14 \%$ de MAT. Les quantités proposées, limitées, couvrent les besoins énergétiques des animaux. Les chèvres sont munies d'une canule du rumen 3 sem avant le début des mesures effectuées en fin de lactation $\left(20^{\circ} \mathrm{sem}\right)$ puis en période de tarissement. Après induction des chaleurs et saillie des chèvres, les mesures sont reprises en $7^{e}, 15^{e}$ et $20^{e}$ sem de gestation, puis en $4^{\mathrm{e}}$ et $8^{\mathrm{e}} \mathrm{sem}$ de lactation. $\dot{A}$ chaque période, sont déterminées successivement les pertes irréversibles de $\mathrm{CO}_{2}$ dans le rumen, après injection intraruminale d'une dose de $80 \mu \mathrm{Ci}$ de ${ }^{14} \mathrm{CO}_{3} \mathrm{HNa}$ (Nolan et Leng, 1972) puis la digestibilité de la ration et le bilan azoté, sur $7 \mathbf{j}$, et enfin l'estimation de la part de l'urée sanguine transférée au rumen par simple injection dans la veine jugulaire de ${ }^{14} \mathrm{C}$ urée $(100 \mu \mathrm{Ci})$ et mesure des pertes irréversibles d'urée dans le sang et du $\mathrm{CO}_{2}$ dans le rumen (Obara et Shimbayiashi, 1987). Cet ensemble de mesures est effectué une seule fois, sur chaque animal et à chaque période.
Résultats et discussion - Les quantités ingérées permettent aux chèvres de couvrir leurs besoins énergétiques (sauf en fin de lactation) et azotés (tableau I). La différence PDIN-PDIE (DPDI), négative quelle que soit la période ( -6 à -12 g/UFL), présente des variations liées à celles de la digestibilité de la matière organique et de la quantité d'azote ingérée. Les urémies moyennes sont minimales au tarissement et en début de gestation (tableau II). Elles sont maximales en fin de gestation et en lactation, quand les apports alimentaires de matières azotées sont les plus élevés. En revanche, les teneurs moyennes en $\mathrm{NNH}_{3}$ dans le jus de rumen sont les plus faibles en fin de gestation et en début de lactation. Les valeurs du rapport (PDIN-PDIE)/UFL n'expliquent pas ces différences.

Les quantités d'urée présentes dans l'organisme, ainsi que celles synthétisées chaque jour, sont maximales en fin de gestation et en lactation. Les quantités moyennes d'urée recyclée dans le rumen varient significativement d'un stade à l'autre. Entre le tarissement et le début de lactation, elles sont multipliées par 5 , alors que les apports d'azote alimentaire ont seulement doublé. La quantité de MOD ingérée est le meilleur prédicteur $(66 \%$ de la variance expliquée) des variations du recyclage d'urée.

$\mathrm{N}$ urée recyclée $(\mathrm{mg} / \mathrm{kgPV} / \mathrm{j})=$ $6,6+0,0002 \cdot \mathrm{MOD}^{2}(\mathrm{~g} / \mathrm{j}), r=0,81, n=28$. 
Le modèle explicatif proposé par Kennedy et Milligan (1980) pour des ovins à l'entretien peut être appliqué sur chèvres en production :

$\mathrm{N}$ urée recyclée $(\mathrm{mg} / \mathrm{kgPV} / \mathrm{j})=$ $259,6+0,09$-urémie $(\mathrm{mg} / \mathrm{l})+0,0001 \cdot$ urémie$^{2}$ $-0,80 \cdot \mathrm{NNH}_{3}(\mathrm{mg} / \mathrm{l})+0,001 \cdot \mathrm{NNH}_{3}{ }^{2}$

$-0,41 \cdot \mathrm{MOD}(\mathrm{g} / \mathrm{j})+0,0004 \cdot \mathrm{MOD}^{2}$, $r=0,83, n=28$.

Cependant, dans nos conditions, les variables urémie et $\mathrm{NNH}_{3}$ ne sont pas significatives $(P>0,05)$. L'étude des résidus fait apparaître un effet significatif du stade physiologique : la part du recyclage non expliquée par les variations de MOD est plus élevée en milieu et fin de gestation et en début de lactation que pendant les autres périodes.

En conclusion, les modifications de l'ingestion et de la digestion des aliments observées aux différents stades physiologiques ne sont pas suffisantes pour expliquer les variations du recyclage d'urée. D'autres facteurs liés strictement au stade physiologique devront être étudiés.

Kennedy PM, Milligan LP (1980) Can J Anim Sci 60, 205-221

Nolan JV, Leng RA (1972) Br J Nutr 27, 177-194

Obara Y, Shimbayiashi K (1987) Jpn J Zootech Sci 58, 611-617

Tableau I. Bilan de l'utilisation de l'azote et de l'énergie.

\begin{tabular}{|c|c|c|c|c|c|c|c|}
\hline & Ingéré & $\begin{array}{l}\text { Azote }(g / j) \\
\text { Urinaire }\end{array}$ & Retenu & $\begin{array}{r}\text { Dig } \\
\text { Azote }\end{array}$ & $\begin{array}{l}\text { tibilité (\%) } \\
\text { Mat organ }\end{array}$ & $\begin{array}{l}\text { Apports énergie } \\
\% \text { besoin }\end{array}$ & $\begin{array}{l}D P D I \\
g / U F L .\end{array}$ \\
\hline \multicolumn{8}{|l|}{ Lactation } \\
\hline $20^{\mathrm{e}} \mathrm{sem}$ & $32,6^{\mathrm{ab}}$ & 14,8 & $2,3^{b}$ & 77,5 & 75,2 & 91 & $-9,1$ \\
\hline $\begin{array}{l}\text { Tarissement } \\
\text { Gestation }\end{array}$ & $21,6^{b}$ & 11,7 & $4,9^{b}$ & 77,1 & 74,2 & 110 & $-11,9$ \\
\hline $\begin{array}{c}7^{\mathrm{e}} \mathrm{sem} \\
15^{\mathrm{e}} \mathrm{sem} \\
20^{\mathrm{e}} \mathrm{sem}\end{array}$ & $\begin{array}{l}21,9^{\mathrm{b}} \\
25,7^{\mathrm{ab}} \\
31,9^{\mathrm{ab}}\end{array}$ & $\begin{array}{l}14,3 \\
10,8 \\
11,1\end{array}$ & $\begin{array}{c}2,6^{b} \\
8,4^{a b} \\
12,8^{a}\end{array}$ & $\begin{array}{l}77,6 \\
75,6 \\
75,2\end{array}$ & $\begin{array}{l}73,5 \\
71,5 \\
71,9\end{array}$ & $\begin{array}{l}106 \\
107 \\
108\end{array}$ & $\begin{array}{r}-12,1 \\
-6,6 \\
-6,3\end{array}$ \\
\hline \multicolumn{8}{|l|}{ Lactation } \\
\hline $\begin{array}{l}4^{e} \text { sem } \\
8^{e} \text { sem } \\
\text { ETR }\end{array}$ & $\begin{array}{c}37,6^{\mathrm{a}} \\
37,9^{\mathrm{a}} \\
7,7\end{array}$ & $\begin{array}{r}15,6 \\
15,3 \\
2,9\end{array}$ & $\begin{array}{l}1,4^{b} \\
6,3^{a b} \\
3,5\end{array}$ & $\begin{array}{r}71,5 \\
74,7 \\
2,2\end{array}$ & $\begin{array}{r}67,2 \\
66,5 \\
2,3\end{array}$ & $\begin{array}{l}100 \\
100\end{array}$ & $\begin{array}{r}-11,5 \\
-8,0 \\
3,1\end{array}$ \\
\hline
\end{tabular}

Tableau II. Métabolisme de l'urée.

\begin{tabular}{|c|c|c|c|c|c|}
\hline & $\begin{array}{l}\text { Urémie } \\
(m g / l)\end{array}$ & $\begin{array}{c}\mathrm{NNH}_{3} \text { jus de rumen } \\
(\mathrm{mgN} / \mathrm{l})\end{array}$ & $\begin{array}{l}\text { Pool urée } \\
(g N / k g P V)\end{array}$ & $\begin{array}{l}\text { Pertes urée } \\
(g N / k g P V / j)\end{array}$ & $\begin{array}{c}\text { Urée recyclée dans rumen } \\
(g N / k g P V / j)\end{array}$ \\
\hline \multicolumn{6}{|l|}{ Lactation } \\
\hline $20^{\mathrm{e}} \mathrm{sem}$ & $309^{b}$ & $167^{\mathrm{b}}$ & $0,08^{b}$ & $0,63^{b}$ & $0,19^{a b}$ \\
\hline $\begin{array}{l}\text { Tarissement } \\
\text { Gestation }\end{array}$ & $275^{b}$ & $218^{b}$ & $0,09^{b}$ & $0,45^{b}$ & $0,05^{b}$ \\
\hline $7^{e} \mathrm{sem}$ & $272^{b}$ & $332^{a}$ & $0,07^{b}$ & $0,41^{b}$ & $0,06^{b}$ \\
\hline $15^{e} \mathrm{sem}$ & $250^{\circ}$ & $238^{\mathrm{b}}$ & $0,07^{b}$ & $0,39^{b}$ & $0,11^{a b}$ \\
\hline $20^{e} \mathrm{sem}$ & $510^{a}$ & $183^{b}$ & $0,15^{a}$ & $0,85^{a}$ & $0,19^{a b}$ \\
\hline \multicolumn{6}{|l|}{ Lactation } \\
\hline $4^{e}$ sem & $330^{\mathrm{b}}$ & $191^{\mathrm{b}}$ & $0,10^{b}$ & $0,63^{\mathrm{b}}$ & $0,26^{a b}$ \\
\hline $8^{e}$ sem & $402^{b}$ & $218^{b}$ & $0,12^{b}$ & $0,63^{b}$ & $0,12^{\mathrm{ab}}$ \\
\hline ETR & 83 & 55 & 0,02 & 0,04 & 0,08 \\
\hline
\end{tabular}

$a, b$ : dans une mème colonne, les valeurs indicées qui n'ont pas une lettre en commun sont significativement différentes $(P<0,05)$. 